\title{
AN ADAPTIVE VARIATIONAL MODEL FOR MEDICAL IMAGES RESTORATION
}

\author{
Tran Thi Thu Thao ${ }^{1}$, Pham Cong Thang ${ }^{2, *}$, Andrei V. Kopylov ${ }^{3}$, Nguyen Van Nguyen ${ }^{2}$ \\ 1 The University of Danang-University of Economics, 71 Ngu Hanh Son, Danang, Viet Nam \\ thaotran@due.udn.vn \\ 2 The University of Danang-University of Science and Technology, 54 Nguyen Luong Bang, Danang, Viet Nam \\ (pcthang, nvnguyen)@dut.udn.vn \\ ${ }^{3}$ Tula State University, 92 Lenin Ave., Tula, Russia \\ And.Kopylov@gmail.com
}

Commission VI, WG VI/4

KEY WORDS: Adaptive model, Medical image denoising, Mixed noise, Total variation, Laplacian regularizer, Split Bregman

\begin{abstract}
:
Image denoising is one of the important tasks required by medical imaging analysis. In this work, we investigate an adaptive variation model for medical images restoration. In the proposed model, we have used the first-order total variation combined with Laplacian regularizer to eliminate the staircase effect in the first-order TV model while preserve edges of object in the piecewise constant image. We also propose an instance of Split Bregman method to solve the proposed denoising model as an optimization problem. Experimental results from mixed Poisson-Gaussian noise are given to demonstrate that our proposed approach outperforms the related methods
\end{abstract}

\section{INTRODUCTION}

In the process of receiving and transmitting through communication channels, the image is usually corrupted by noise of a different nature. Therefore, image restoration and, in particular, denoising is the most fundamental tasks of medical image processing to help supply a better diagnosis and treatment. An important requirement for the image denoising procedure is to preserve local image features for efficient subsequent analysis. Image restoration is often formulated as the problem of reconstruction of a true image, damaged by some noise, from the observed image of the same size. The sought-for image is a solution of the corresponding inverse problem (Pham, 2015, Pham, 2016).

Many types of noise have been studied in the literature, but the majority of models in image denoising are associated with additive Gaussian noise and Poisson noise (Bertero et al., 2009, Pham, 2017, Pham, 2018). One of the most common variational models to restore an image is a well-known Rudin, Osher and Fatemi model (ROF model), which allows to remove Gaussian noise effectively (Rudin et al., 1992). The authors in (Le et al., 2007) had proposed total-variation regularized denoising model along the lines of ROF model but modified for use with Poisson noise(M-ROF model). However, the considered models ROF and M-ROF are effective for image reconstruction, if there is either Gaussian or Poisson noise. In practical, image can be corrupted by different types of noise like as mixed Poisson-Gaussian noise (Luisier, 2011, Lanza et al., 2014, Jezierska et al., 2014). The linear combination of models ROF and M-ROF was proposed for mixed Poisson-Gaussian in (De los Reyes, Schnlieb, 2013):

$$
u^{*}=\underset{u \in B V(\Omega)}{\arg \min } E(u)
$$

\footnotetext{
${ }^{*}$ Corresponding author
}

$$
\begin{aligned}
E(u) & =\int_{\Omega}|\nabla u| d x+\frac{\lambda}{2} \int_{\Omega}(u-f)^{2} d x \\
& +\beta \int_{\Omega}(u-f \log u) d x
\end{aligned}
$$

where $\quad f(x)$ - the observed image

$x=\left(x_{1}, x_{2}\right) \in \Omega$

$\Omega \in \mathbb{R}^{2}$ - an open bounded domain

$B V$ - the space of functions of bounded variation

$\int_{\Omega}|\nabla u| d x-$ total variation of $u$

$\lambda, \beta$ - positive regularization parameters

The model (1) can denoise an image corrupted by a mixture of Poisson and Gaussian noise. However, problems of negative values arise in the numerical algorithms. To avoid this problem, authors in (Pham et al., 2018) proposed a modified scheme of gradient descent (MSGD) that is impossible to avoid sign-changing of the solution during the optimization process and guarantees the reconstructed image to be positive in the image domain. The authors proposed a time marching strategy to the associated Euler-Lagrange equation. The algorithm has the advantage of being rather easy to implement and to offer a good compromise between performance and quality of results. Like similar algorithms, no previous training is required (by contrast with deep learning techniques), and only one observation of the image is needed. However, this method is slow due to the constraint of stability conditions about the time step size. Hence, construction of fast and effective algorithms has become an active research area so far. The model (1) performs well in preserving image edges compared with other related method. However, this model has sometimes undesirable staircase effect in some cases, namely, the transformation of smooth regions into piecewise constants regions (Chan et al., 2015). 
In this paper, we proposed adaptive model in which the first-order TV is combined with Laplacian regularizer to eliminate the staircase effect in the first-order TV model (1) while preserve edges of object in the piecewise constant image. Then, we employ split Bregman method (SBM) to solve correspondent optimization problem (Goldstein, 2009). Experimental results show the effectiveness of the proposed method for denoising medical images corrupted by mixed Poisson-Gaussian noise. Comparison with other related methods and state-of-the-art algorithms is provided numerically as well.

\section{THE PROPOSED NEW MODEL AND ALGORITHM}

\subsection{Adaptive variational model}

In this work, we focus on the model (1) and propose the following adaptive model:

$$
\begin{aligned}
& u^{*}=\underset{u \in T(\Omega)}{\arg \min } E(u) \\
& E(u)=\alpha \int_{\Omega}|\nabla u| d x+\mu \int_{\Omega}|\triangle u| d x \\
& \frac{\lambda}{2} \int_{\Omega}(u-f)^{2} d x+\beta \int_{\Omega}(u-f \log u) d x
\end{aligned}
$$

where $f$ - the observed image

$x=\left(x_{1}, x_{2}\right) \in \Omega$

$\Omega \in \mathbb{R}^{2}$ - an open bounded domain

$T(\Omega)=\{u \in B V(u): u>0\}$

$\lambda, \beta$ - positive regularization parameters

$\triangle u$ - Laplacian operator of $u$

$\mu$ and $\alpha$ - constant function

In recent years, many efficient numerical algorithms have emerged (Chambolle, 2004, Wang et al., 2008, Goldstein, 2009, Pham et al., 2017), which can be used to solve the proposed model (2). In this paper, we decide to employ split Bregman method (Goldstein, 2009) to solve our minimization problem (2). In next subsection, we present the Split-Bregman method which is used solve the energy minimization problem (2).

\subsection{Split-Bregman method}

The split Bregman method performs break the minimization problem down into easy subproblems (Goldstein, 2009, Getreuer, 2012). Subproblems can be directly solved with tools like Fast Fourier Transform, shrinkage operator that makes the optimization algorithm rather fast. We have a scalar $\gamma$ and two convex functionals $\Psi(\cdot)$ and $G(\cdot)$; and that we need to solve the following constrained optimization problem:

$$
\text { find } \underset{u, d}{\arg \min }\|d\|_{1}+\frac{\gamma}{2} G(u), \text { s.t. } d=\Psi(u)
$$

We convert (3) into an unconstrained problem:

$$
\text { find } \underset{u, d}{\arg \min }\|d\|_{1}+\frac{\gamma}{2} G(u)+\frac{\rho}{2}\|d-\Psi(u)-b\|_{2}^{2}
$$

where $\rho$-penalty parameter (a positive constant)

$b$ - variable of the Bregman iterations

$d$ - splitting variable
The solution to problem (4) can be approximated by the Split Bregman Algorithm:

$$
\begin{aligned}
& u^{(k+1)}=\underset{u}{\arg \min } \frac{\gamma}{2} G(u)+\frac{\rho}{2}\left\|d^{(k)}-\Psi(u)-b^{(k)}\right\|_{2}^{2} \\
& d^{(k+1)}=\underset{d}{\arg \min }\|d\|_{1}+\frac{\rho}{2}\left\|d-\Psi\left(u^{(k+1)}\right)-b^{(k)}\right\|_{2}^{2} \\
& b^{(k+1)}=b^{(k)}+\Psi\left(u^{(k+1)}\right)-d^{(k+1)}
\end{aligned}
$$

\subsection{Algorithm for the proposed model}

Now we return to the problem (2). We introduce three new variables and rewrite (2) in the constrained optimization problem as follows:

$$
\begin{array}{r}
\underset{u, d, r}{\arg \min }\left(\alpha\|d\|_{1}+\mu\|r\|_{1}+\frac{\gamma}{2} G(z)\right. \\
\text { s.t. } d=\nabla u, z=u, r=\triangle u
\end{array}
$$

where

$$
G(z)=\frac{\lambda}{2}(z-f)^{2}+\beta(z-f \log z)
$$

The augmented Lagrangian functional for the constrained optimization problem (5) is defined as:

$$
\begin{aligned}
\underset{u, d, r}{\arg \min }( & \alpha\left\|\left(d_{1}, d_{2}\right)\right\|_{1}+\mu\|r\|_{1}+\frac{\gamma}{2} G(z)+ \\
& +\frac{\rho_{1}}{2}\left\|z-u-b_{z}\right\|_{2}^{2}+\frac{\rho_{2}}{2}\left\|d_{1}-\nabla_{1} u-b_{1}\right\|_{2}^{2}+ \\
& \left.+\frac{\rho_{2}}{2}\left\|d_{2}-\nabla_{2} u-b_{2}\right\|_{2}^{2}+\frac{\rho_{3}}{2}\left\|r-\Delta u-b_{3}\right\|_{2}^{2}\right)
\end{aligned}
$$

where $\rho_{1}, \rho_{2}, \lambda, \gamma$ - positive parameters

$b_{z}, b_{i},(i=1 . .3)$ - variables of the Bregman iterations

$\left(\nabla_{1} u, \nabla_{2} u\right)$ - spatial derivatives

$\left(d_{1}, d_{2}\right)$ - splitting variables

The discrete gradient $\nabla u$ and Laplacian operator $\triangle u$ of an image $u$ for the pixel at coordinates $(i, j)$ in $u(i=1 . . M ; k=$ $1 . . N)$ are defined like:

$$
\begin{aligned}
& \nabla u_{j, k}=\left(\nabla_{1} u(i, j), \nabla_{2} u(i, j)\right) \\
& \nabla_{1} u(i, j)=u(i+1, j)-u(i-1, j) \\
& \nabla_{2} u(i, j)=u(i, j+1)-u(i, j-1) \\
& |\nabla u|=\sqrt{\left(\nabla_{1} u(i, j)\right)^{2}+\left(\nabla_{2} u(i, j)\right)^{2}} \\
& \triangle u(i, j)=-4 u(i, j)+u(i+1, j) \\
& +u(i-1, j)+u(i, j+1)+u(i, j-1)
\end{aligned}
$$

The split Bregman method for solving (6) is described as follows:

$$
\begin{aligned}
& u^{(k+1)}=\underset{u}{\arg \min } \frac{\rho_{1}}{2}\left\|z-u-b_{z}\right\|_{2}^{2}+\frac{\rho_{2}}{2}\left\|d_{1}-\nabla_{1} u-b_{1}\right\|_{2}^{2} \\
& +\frac{\rho_{2}}{2}\left\|d_{2}-\nabla_{2} u-b_{2}\right\|_{2}^{2}+\frac{\rho_{3}}{2}\left\|r-\triangle u-b_{3}\right\|_{2}^{2}
\end{aligned}
$$




$$
\begin{aligned}
& z^{(k+1)}=\underset{z}{\arg \min } \frac{\gamma}{2} G(z)+\frac{\rho_{1}}{2}\left\|z-u^{(k+1)}-b_{z}^{(k)}\right\|_{2}^{2} \\
& d_{1}^{(k+1)}=\underset{d}{\arg \min } \alpha\left\|d_{1}\right\|_{1}+\frac{\rho_{2}}{2}\left\|d_{1}-\nabla_{1} u^{(k+1)}-b_{1}^{(k)}\right\|_{2}^{2} \\
& d_{2}^{(k+1)}=\underset{d}{\arg \min } \alpha\left\|d_{2}\right\|_{1}+\frac{\rho_{2}}{2}\left\|d_{2}-\nabla_{2} u^{(k+1)}-b_{2}^{(k)}\right\|_{2}^{2} \\
& r^{(k+1)}=\underset{r}{\arg \min } \mu\|r\|_{1}+\frac{\rho_{3}}{2}\left\|r-\triangle u^{(k+1)}-b_{3}^{(k)}\right\|_{2}^{2} \\
& b_{z}^{(k+1)}=b_{z}^{(k)}+u^{(k+1)}-z^{(k+1)} \\
& b_{1}^{(k+1)}=b_{1}^{(k)}+\nabla_{1} u^{(k+1)}-d_{1}^{(k+1)} \\
& b_{2}^{(k+1)}=b_{2}^{(k)}+\nabla_{2} u^{(k+1)}-d_{2}^{(k+1)} \\
& b_{3}^{(k+1)}=b_{3}^{(k)}+\triangle u^{(k+1)}-r^{(k+1)}
\end{aligned}
$$

There are four subproblems to solve: $u, z, d$ and $r$.

Subproblem 1. The $u$ subproblem is a quadratic optimization problem, whose optimality condition reads:

$$
\begin{aligned}
& \rho_{1}\left(u-z+b_{z}\right)+\rho_{2} \nabla_{1}^{T}\left(\nabla_{1} u+b_{1}-d_{1}\right) \\
+ & \rho_{2} \nabla_{2}^{T}\left(\nabla_{2} u+b_{2}-d_{2}\right)+\rho_{3} \triangle\left(\triangle u+b_{3}-r\right)=0
\end{aligned}
$$

We can rewrite the equation (7) as:

$$
\begin{aligned}
& \left(\rho_{1}+\rho_{2}\left(\nabla_{1}^{T} \nabla_{1}+\nabla_{2}^{T} \nabla_{2}\right)+\rho_{3}(\triangle(\triangle))\right) u^{(k+1)} \\
= & \rho_{1}\left(z^{(k)}-b_{z}^{(k)}\right)+\rho_{2} \nabla_{1}^{T}\left(d_{1}^{(k)}-b_{1}^{(k)}\right) \\
+ & \rho_{2} \nabla_{2}^{T}\left(d_{2}^{(k)}-b_{2}^{(k)}\right)+\rho_{3} \triangle\left(r^{(k)}-b_{3}^{(k)}\right)
\end{aligned}
$$

under considering periodic boundary conditions. Note that left-hand-side matrix in (8) includes a Laplacian matrix $\left(\nabla_{1}^{T} \nabla_{1}+\nabla_{2}^{T} \nabla_{2}=-\Delta\right)$ and is strictly diagonally dominant. Following (Tao et al., 2009, Tai et al., 2011), equation (8) can be solved efficiently with one Fourier transform operation and one inverse FFT operation as:

$$
\begin{gathered}
u^{(k+1)}=\mathcal{F}^{-1}\left(\frac{\mathcal{F}(q)}{\rho_{1}-\rho_{2} \mathcal{F}(\Delta)+\rho_{2} \mathcal{F}(\triangle(\triangle))}\right) \\
\text { with } q=\rho_{1}\left(z^{(k)}-b_{z}^{(k)}\right)+\rho_{2} \nabla_{1}^{T}\left(d_{1}^{(k)}-b_{1}^{(k)}\right) \\
+\rho_{2} \nabla_{2}^{T}\left(d_{2}^{(k)}-b_{2}^{(k)}\right)+\rho_{3} \triangle\left(r^{(k)}-b_{3}^{(k)}\right)
\end{gathered}
$$

where $\mathcal{F}$ and $\mathcal{F}^{-1}$ are the forward and inverse Fourier transform operators.

Subproblem 2. The optimality condition for the $z$ subproblem is given by:

$$
\frac{\gamma}{2}\left(\lambda(z-f)+\beta\left(1-\frac{f}{z}\right)\right)+\rho_{1}\left(z-u^{(k+1)}-b_{z}^{(k)}\right)=0
$$

This equation can be rewritten as:

$$
\begin{aligned}
& \left(\frac{\gamma}{2} \lambda+\rho_{1}\right)\left(z^{(k+1)}\right)^{2} \\
- & \left(\frac{\gamma}{2} \lambda f-\beta \frac{\gamma}{2}+\rho_{1}\left(u^{(k+1)}+b_{z}^{(k)}\right)\right) z^{(k+1)}-\frac{\gamma}{2} \beta f=0
\end{aligned}
$$

The positive solution is given by:

$$
z^{(k+1)}=W^{(k)}+\sqrt{\left(W^{(k)}\right)^{2}+\frac{\gamma \beta f}{\gamma \lambda+2 \rho_{1}}}
$$

where

$$
W^{(k)}=\frac{\lambda \gamma f-\beta \gamma+2 \rho_{1}\left(z^{(k+1)}+b_{z}^{(k)}\right)}{2\left(\gamma \lambda_{1}+2 \rho_{1}\right)}
$$

Subproblem 3. The solution of the $d=\left(d_{1}, d_{2}\right)$ subproblem can readily be obtained by applying the soft thresholding operator (Micchelli et al., 2011). We can use shrinkage operators to compute the optimal values of $d_{1}$ and $d_{2}$ separately:

$$
\begin{aligned}
& d_{i}^{(k+1)}=\operatorname{shrink}\left(\nabla_{i} u^{(k+1)}+b_{i}^{(k)}, \frac{\alpha}{\rho_{2}}\right) \\
& =\frac{\nabla_{i} u^{(k+1)}+b_{i}^{(k)}}{\left|\nabla_{i} u^{(k+1)}+b_{i}^{(k)}\right|} \cdot \max \left(\left|\nabla_{i} u^{(k+1)}+b_{i}^{(k)}\right|-\frac{\alpha}{\rho_{2}}, 0\right)
\end{aligned}
$$

Subproblem 4. The solution of the $r$ subproblem can be obtained by applying the soft thresholding operator too:

$$
\begin{aligned}
& r^{(k+1)}=\operatorname{shrink}\left(\triangle u^{(k+1)}+b_{3}^{(k)}, \frac{\mu}{\rho_{3}}\right) \\
& =\frac{\triangle u^{(k+1)}+b_{3}^{(k)}}{\left|\triangle u^{(k+1)}+b_{3}^{(k)}\right|} \cdot \max \left(\left|\triangle u^{(k+1)}+b_{3}^{(k)}\right|-\frac{\mu}{\rho_{3}}, 0\right)
\end{aligned}
$$

The algorithm. The complete method is summarized in Algorithm (1). We need a stopping criterion for the iteration; we end the loop if the maximum number of allowed outer iterations $N$ has been carried out (to guarantee an upper bound on running time) or the following condition is satisfied for some prescribed tolerance $\varsigma$ :

$$
\frac{\left\|u^{(k)}-u^{(k-1)}\right\|_{2}}{\left\|u^{(k)}\right\|_{2}}<\varsigma
$$

where $\varsigma$ is a small positive parameter. For our experiments, we set tolerance in $(13) \varsigma=0.0005$ and $N=200$.

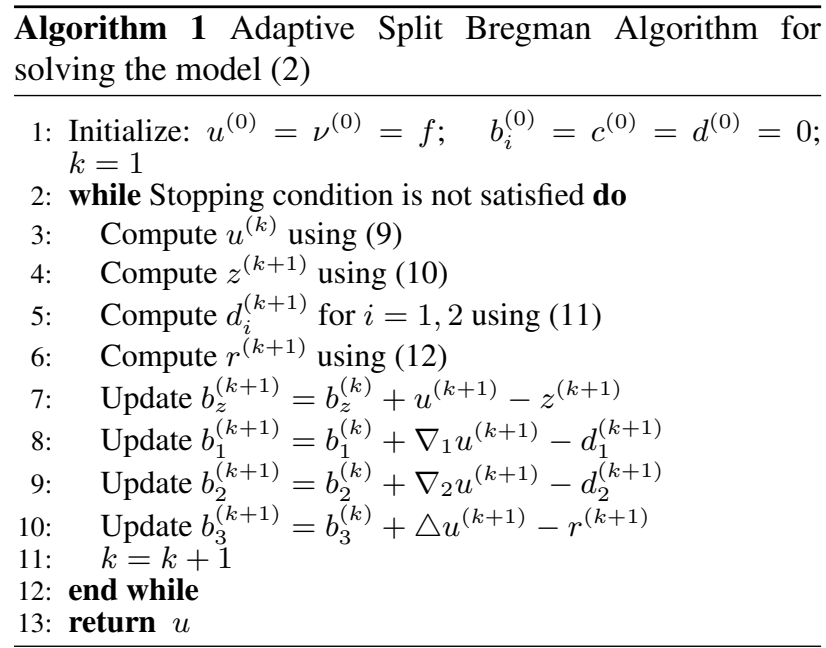

\section{EXPERIMENTAL RESULTS}

In this section, we demonstrate our algorithm for staircasing reduction in medical image denoising. All simulations are performed in Matlab 7.8 on a PC with an Intel Core (TM) i7 $\mathrm{CPU}$ at $1.99 \mathrm{GHz}$ and $8.00 \mathrm{~GB}$ of memory. Test images are 
Magnetic resonance imaging (MRI) ${ }^{1}$ scans with size $360 \times$ 360. Noisy observation is generated by Poisson noise with peak $I_{\max }$, and by Gaussian noise with standard deviation $\sigma_{G}$ to the test images. To quantify the denoising results, we use the Peak-Signal-to-Noise Ratio (PSNR) and the Structural Similarity Index (SSIM) defined as (Bovik, 2006):

$$
\begin{gathered}
P S N R=10 \log _{10}\left(\frac{M N I_{\max }^{2}}{\left\|u^{*}-u\right\|_{2}^{2}}\right) \\
\operatorname{SSIM}\left(u, u^{*}\right)=\frac{\left(2 \mu_{u} \mu_{u^{*}}+c_{1}\right)\left(2 \sigma_{u, u^{*}}+c_{2}\right)}{\left(\mu_{u}^{2}+\mu_{u^{*}}^{2}+c_{1}\right)\left(\sigma_{u}^{2}+\sigma_{u^{*}}^{2}+c_{2}\right)}
\end{gathered}
$$

where $u, u^{*}$ are the original image, the reconstructed or noisy image accordingly; $I_{\max }$ is the maximum intensity of the original image; $M$ and $N$ are the number of image pixels in rows and columns; $\mu_{u}, \mu_{u^{*}}-$ means of images; $\sigma_{u}, \sigma_{u^{*-}}$ standard deviations (the square root of variance) of images; $\sigma_{u, u^{*}-}$ covariance of two images $u$ and $u^{*} ; c_{1}=\left(K_{1} L\right)^{2}$, $c_{2}=\left(K_{2} L\right)^{2}, L$ is the dynamic range of the pixel values $(255$ for 8-bit grayscale images), and $K_{1} \ll 1, K_{2} \ll 1$ are small constants.

On Fig. (1) and (2) example results of the proposed method on test images with various noise levels are shown.

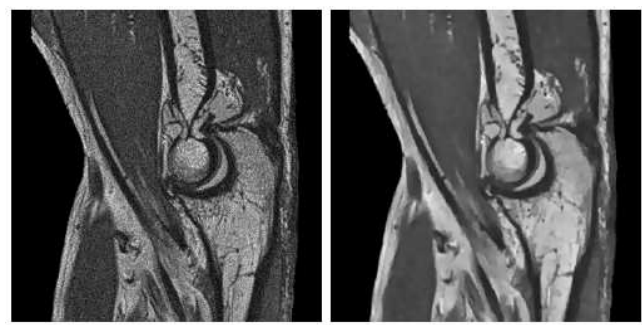

(a)

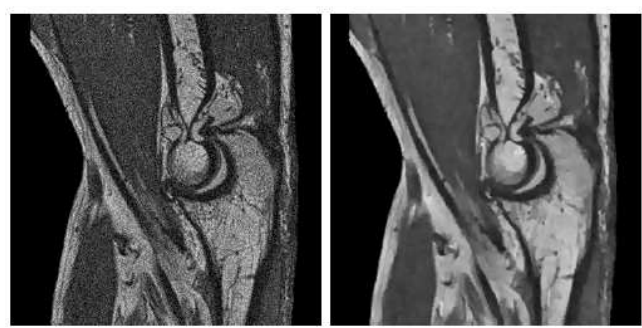

(b)
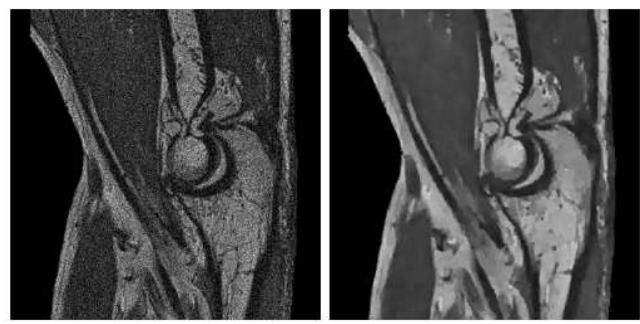

(c)

Figure 1. Image Elbow. Recovered images of our method for removing mixed Poisson-Gaussian noise. From left to right: the noisy image and its recovered image with noise level: (a) $I_{\max }=120, \sigma_{G}=5$; (b)

$$
I_{\max }=120, \sigma_{G}=10 \text {; (c) } I_{\max }=60, \sigma_{G}=5
$$

\footnotetext{
${ }^{1}$ Clinical Images, https://www.healthcare.siemens.co. uk/magnetic-resonance-imaging/magnetom-world/toolkit/ clinical-images, accessed 20/12/2018.
}

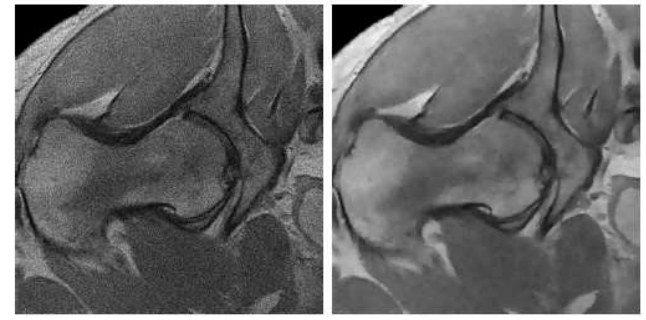

(a)
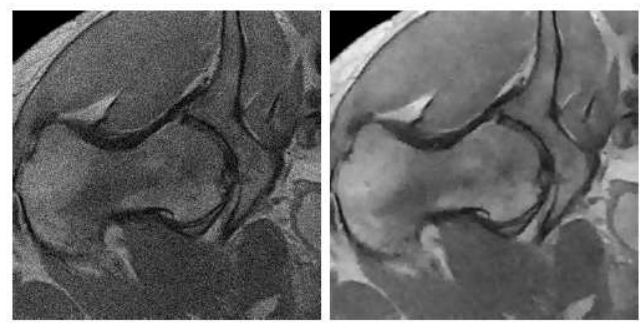

(b)
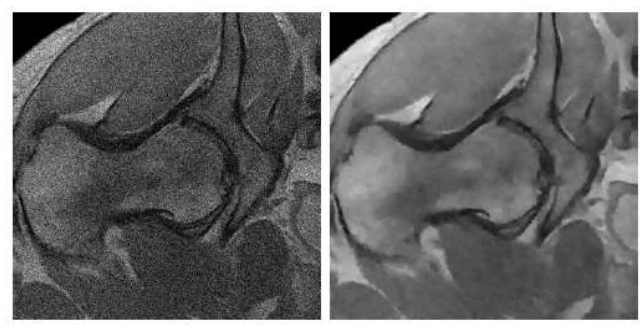

(c)

Figure 2. Image Hip. Recovered images of our method for removing mixed Poisson-Gaussian noise. From left to right: the noisy image and its recovered image with noise level : (a) $I_{\max }=120, \sigma_{G}=5$; (b)

$$
I_{\max }=120, \sigma_{G}=10 \text {; (c) } I_{\max }=60, \sigma_{G}=5
$$

Fig. (3) and (5) show original test images and their noisy images with noise level $I_{\max }=60$ and $\sigma_{G}=10$, used in experimental comparisons. We compare our proposed method with state-of-the-art denoising methods such as SBM (Goldstein, 2009), MSGD (Pham et al., 2018). All of the compared methods perform image denoising with their default parameters. For our proposed method, we set $\lambda=0.4, \beta=$ $0.6, \rho_{2}=0.8, \rho_{3}=1, \alpha=4, \mu=1$. The parameters $\gamma$ and $\rho_{1}$ are chosen to give the best PSNR performance.

Fig. (4) and (6) show recovered results of the compared methods for the test images corrupted by noise with parameters $I_{\max }=60$ and $\sigma_{G}=10$. We can easily detect staircase effect in the mehods SBM and MSGD. It is clear to see that staircase has been successfully reduced in the results by our method.

The PSNR and SSIM values of different methods are displayed in Tables (1) and (2). The best values among all the methods are shown in bold. We can see that the PSNR and SSIM values of our method are better than those of SBM and MSGD on different noise levels in most cases. Experiments demonstrate that the proposed model outperforms relative methods for mixed Poison-Gaussian removal

\section{CONCLUSION}

In this paper, we propose an adaptive variational model for medical images corrupted with a mixed Poisson-Gaussian 
(a)

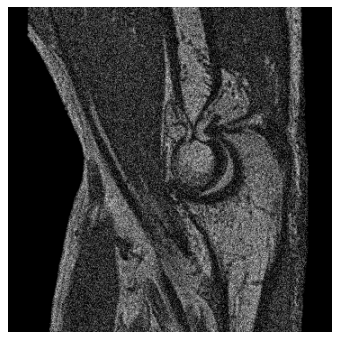

(c)

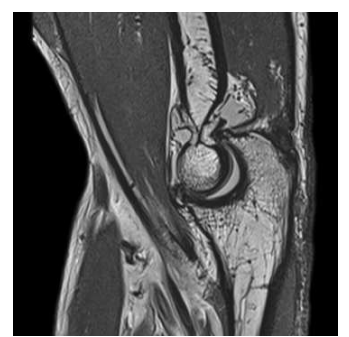

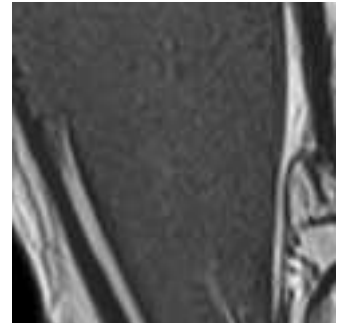

(b)

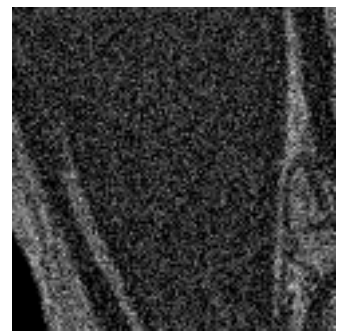

(d)
Figure 3. Image Elbow: (a) Original image, (b) the zoomed-in part of the original image, (c) noisy image, (d) the zoomed-in part of the noisy image

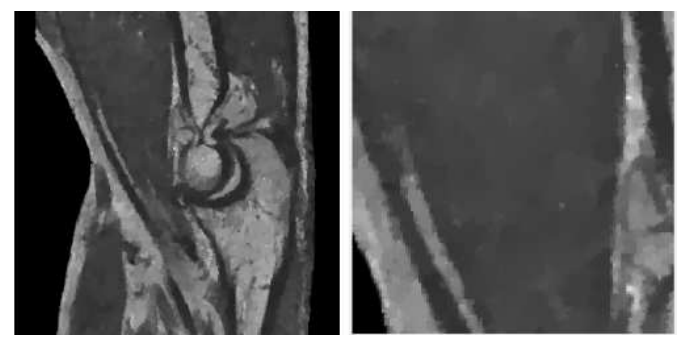

SBM

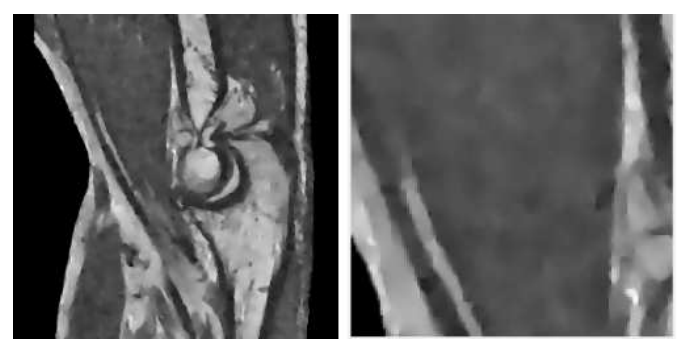

MSGD

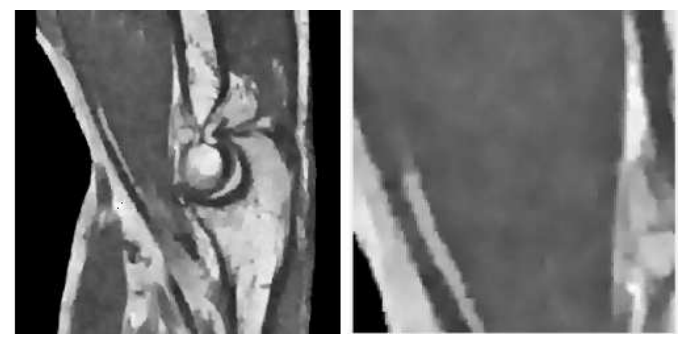

Our proposed

Figure 4. Recovered results of the compared methods for the Elbow Image. From left to right: the restored images and their the zoomed-in part

noise. The new model not only removes noise on image well, but also reduces the staircase effect. In addition, we give an effective algorithm for the proposed model which allows to

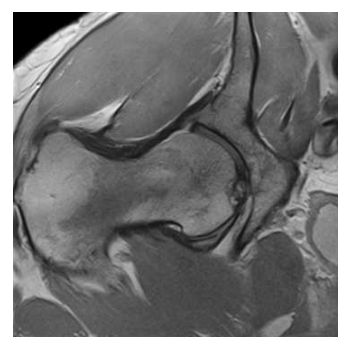

(a)

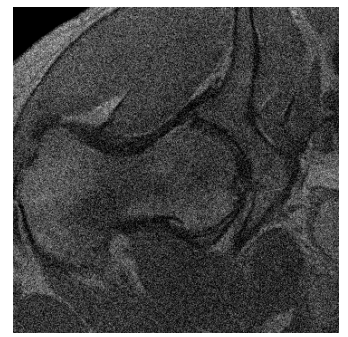

(c)

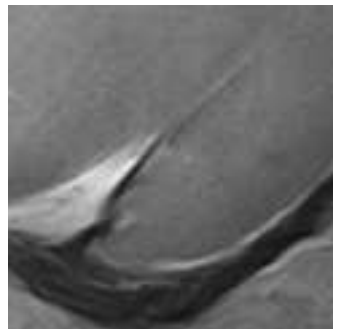

(b)

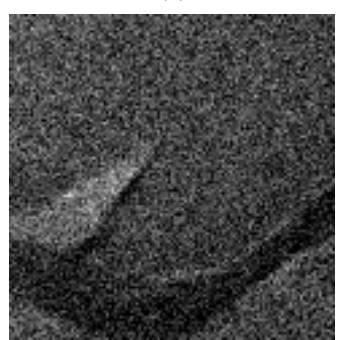

(d)
Figure 5. Image 'Hip': (a) Original image, (b) the zoomed-in part of the original image, (c) noisy image, (d) the zoomed-in part of the noisy image
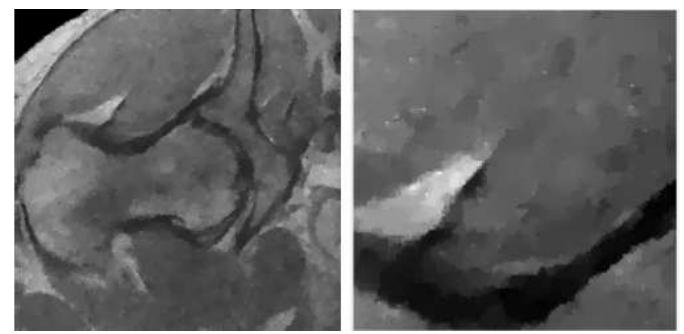

SBM
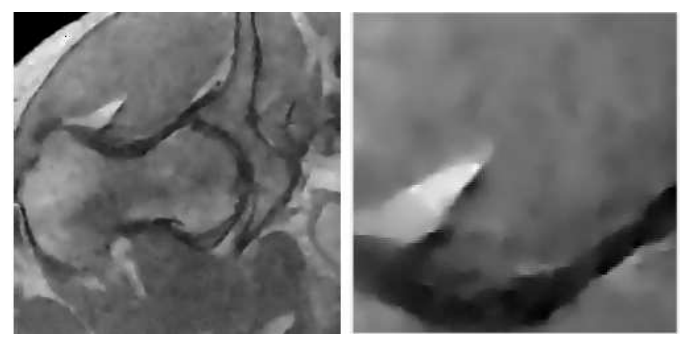

MSGD
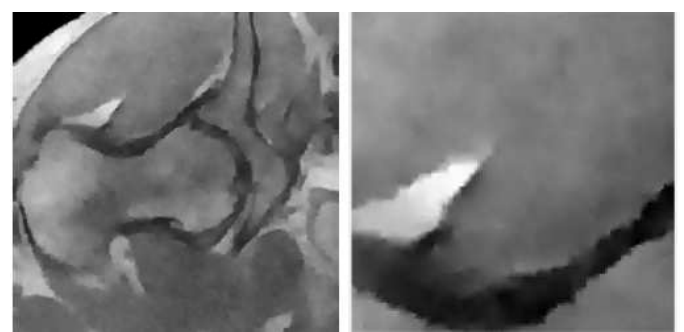

Our proposed

Figure 6. Recovered results of the compared methods for the Hip Image. From left to right: the restored images and their the zoomed-in part

obtain results outperforming the related methods. Our future research will consider the application of the proposed model to mixed Poisson-Gaussian colour image noise removal. 


\begin{tabular}{|c|c|c|c|c|c|}
\hline \multirow{2}{*}{ Image } & \multicolumn{2}{|c|}{ Noisy } & \multirow{2}{*}{ SBM } & \multirow{2}{*}{ MSGD } & \multirow{2}{*}{ Our proposed } \\
\hline & $I_{\max }$ & $\sigma_{G}$ & & & \\
\hline \multirow{4}{*}{ Elbow } & \multirow{2}{*}{120} & 5 & 26.8681 & 27.4881 & 28.2132 \\
\hline & & 10 & 26.4459 & 27.1037 & 27.4336 \\
\hline & \multirow{2}{*}{60} & 5 & 25.8111 & 25.3611 & 27.0221 \\
\hline & & 10 & 24.6620 & 24.8153 & 25.5741 \\
\hline \multirow{4}{*}{ Hip } & \multirow{2}{*}{120} & 5 & 29.3330 & 31.1894 & 31.5953 \\
\hline & & 10 & 29.2205 & 30.0327 & 30.3408 \\
\hline & \multirow{2}{*}{60} & 5 & 28.3415 & 27.9522 & 29.4010 \\
\hline & & 10 & 26.9792 & 27.4590 & 27.9816 \\
\hline
\end{tabular}

Table 1. PSNR values for recovered images given by the compared methods with various levels

\begin{tabular}{|c|c|c|c|c|c|}
\hline \multirow{2}{*}{ Image } & \multicolumn{2}{|c|}{ Noisy } & \multirow{2}{*}{ SBM } & \multirow{2}{*}{ MSGD } & \multirow{2}{*}{ Our proposed } \\
\hline & $I_{\max }$ & $\sigma_{G}$ & & & \\
\hline \multirow{4}{*}{ Elbow } & \multirow{2}{*}{120} & 5 & 0.8111 & 0.8264 & 0.8548 \\
\hline & & 10 & 0.8029 & 0.8217 & 0.8303 \\
\hline & \multirow{2}{*}{60} & 5 & 0.7927 & 0.7605 & 0.8019 \\
\hline & & 10 & 0.7623 & 0.7456 & 0.7716 \\
\hline \multirow{4}{*}{ Hip } & \multirow{2}{*}{120} & 5 & 0.8010 & 0.8335 & $\mathbf{0 . 8 5 0 7}$ \\
\hline & & 10 & 0.7984 & 0.8115 & 0.8171 \\
\hline & \multirow{2}{*}{60} & 5 & 0.7836 & 0.7677 & 0.8015 \\
\hline & & 10 & 0.7367 & 0.7529 & $\mathbf{0 . 7 5 7 9}$ \\
\hline
\end{tabular}

Table 2. SSIM values for recovered images given by the compared methods with various levels

\section{ACKNOWLEDGEMENTS}

The authors would like to thank the anonymous referees for their helpful feedback. This work was supported by The University of Danang, University of Science and Technology, Danang, VietNam, code number of project: T2019-02-02.

\section{REFERENCES}

Bertero, M.and Boccacci, P., Desider, G., Vicidomini, G., 2009. Image deblurring with Poisson data: from cells to galaxies. Inverse Problems, 25 (12):123006.

Bovik, A.C., Wang Z., 2006. Modern Image Quality Assessment (Synthesis Lectures on Image, Video, and Multimedia Processing). Morgan \& Claypool Publishers, 156 pages.

Chambolle, A., 2004. An algorithm for total variation minimization and applications. Journal of Mathematical Imaging and Vision, 26 (6), 89-97.

Chan, R.H., Liang, H., Wei, S., Nikolova, M., Tai, X.C., 2015. High-order total variation regularization approach for axially symmetric object tomography from a single radiograph. Inverse Problems and Imaging, 9(1), 55-77.

De los Reyes, J.C., Schnlieb, C.B., 2013. Image denoising: Learning the noise model via nonsmooth PDE-constrained optimization. Inverse Problems and Imaging, 7 (4), 1183-1214.

Getreuer, P., 2012. Total Variation Deconvolution using Split Bregman. Image Processing On Line, 2, 158-174.

Goldstein, T., Osher S., 2009. The split Bregman method for L1-regularized problems. SIAM Journal on Imaging Sciences, $1,323-343$.

Jezierska, A., Chaux, C., Pesquet, J., Talbot, H., Engler, G., 2014. An EM Approach for Time-Variant Poisson-Gaussian
Model Parameter Estimatio. IEEE Transactions on Signal Processing, 62(1), 17-30.

Lanza, A., Morigi, S., Sgallari, F., Wen, Y.W., 2014. Image restoration with Poisson-Gaussian mixed noise. Computer Methods in Biomechanics and Biomedical Engineering: Imaging and Visualization, 2(1), 257-263.

Le, T., Chartrand, R., Asaki, T. J., 2007. A variational approach to reconstructing images corrupted by Poisson noise. Journal of Mathematical Imaging and Vision, 27, 257-263.

Luisier, F.and Blu, T.and Unser M., 2011. Image denoising in mixed Poisson-Gaussian noise. IEEE Transactions on Image Processing, 20 (3), 696-708.

Micchelli, C.A., Shen, L., Xu, Y., 2011. Proximity algorithms for image models: denoising. Inverse Problems, 27:045009.

Pham, C.T., 2017. Image Processing Procedures Based on Multi-Quadratic Dynamic Programming. Informatica, 41, 255-256.

Pham, C.T., Gamard, G., Kopylov, A., Tran, T.T.T., 2018. An algorithm for image restoration with mixed noise using total variation regularization. Turkish Journal of Electrical Engineering and Computer Sciences, 26 (6), 2831-2845.

Pham, C.T., Kopylov A., 2018. Tree-serial parametric dynamic programming with flexible prior model for image denoising. Computer Optics, 42 (5), 838-845.

Pham, C.T., Kopylov A.V., 2015. Multi-quadratic dynamic programming procedure of edge-preserving denoising for medical images. Int. Arch. Photogramm. Remote Sens. Spatial Inf. Sci., XL-5/W6, 101-106.

Pham, C.T., Kopylov A.V., 2016. Parametric procedures for image denoising with flexible prior model. SoICT '16 Proceedings of the Seventh Symposium on Information and Communication Technology, 294-301.

Pham, C.T., Kopylov, A.V., Dvoenko, S.D., 2017. Edge-preserving denoising based on dynamic programming on the full set of adjacency graphs. Int. Arch. Photogramm. Remote Sens. Spatial Inf. Sci., XLII-2/W4, 55-60.

Rudin, L., Osher, S., Fatemi, E., 1992. Nonlinear total variation-based noise removal algorithms. Physica $D, 60$, 259-268.

Tai, X.C., Hahn, J., Chung, G.J., 2011. A fast algorithm for euler's elastica model using augmented lagrangian method. SIAM Journal on Imaging Sciences, 4(1), 313-344.

Tao, M., Yang, J., He, B., 2009. Alternating direction algorithms for total variation deconvolution in image reconstruction. Research report, Rice University.

Wang, Y.and Yang, J., Yin, W., Zhang, Y., 2008. A new alternating minimization algorithm for total variation image reconstruction. SIAM Journal on Imaging Sciences, 1, 248-272.

Revised March 2019 